\title{
INTENSIDAD EN LOS CAMBIOS DE USOS DEL SUELO REGISTRADOS EN LA COMUNIDAD DE MADRID DURANTE LOS AÑOS 1982 Y 2006
}

\author{
Marta Gallardo \\ Departamento de Geografía. Universidad de Murcia \\ marta.gallardo@um.es
}

\section{RESUMEN}

Se realiza un análisis jerárquico de intensidad de cambios de usos del suelo, a nivel de intervalo de tiempo, a nivel de categorías y a nivel de transiciones, aplicado a la Comunidad de Madrid para los años 1982, 1990, 2000 y 2006. Se observan los principales procesos de cambio y qué categorías se han mostrado estables. Las zonas de extracción minera, vertederos y en construcción son las más intensas, en pérdidas y en ganancias. La artificialización del territorio está relacionada con el aumento de la accesibilidad por infraestructuras de transporte y con la pérdida de superficie agrícola.

Palabras clave: cambios usos del suelo; análisis de intensidad; SIG; crecimiento urbano; Madrid.

\section{ABSTRACT}

A hierarchical analysis of intensity of land use changes, at time interval level, category level and transition level, is applied to the Region of Madrid for the years 1982, 1990, 2000 and 2006. Main processes of change and land use categories that have been stable are shown. Mining, landfill and areas under construction are the most intense, in losses and in gains. Artificialization of the territory is related to the increase of the accessibility by transport infrastructures and with the loss of agricultural areas.

Keywords: land use change; intensity analysis; GIS; urban growth; Madrid.

Fecha de recepción: abril 2016.

Fecha de aceptación: mayo 2017. 


\section{INTRODUCCIÓN}

En las últimas décadas la Comunidad de Madrid ha experimentado un proceso de artificialización, registrando altas tasas de urbanización del territorio (López de Lucio, 2003; Naredo y García-Zaldivar, 2008). Este aumento del uso urbano está relacionado con una mayor accesibilidad, asociada a un aumento de la infraestructura vial (Gago et al., 2004; García Palomares y Gutiérrez Puebla, 2007), pero también con un abandono de las tierras agrícolas (Plata Rocha et al., 2009; Ruiz-Benito et al., 2010). La artificialización del territorio conlleva problemas potenciales como pueden ser la degradación y fragmentación de paisajes (Alig et al., 2003), aumento de la ocurrencia de incendios forestales (Gallardo et al., 2016), aparición de islas de calor (Zhang, et al., 2013), mayor emisión de gases invernadero y otros contaminantes (Chen, 2002; Peña et al., 2007), presión urbanística cerca de las áreas naturales protegidas o incluso en su interior (Mallinis et al., 2011; Pérez-Hugalde et al., 2011; Hewitt et al., 2016; Gallardo y Martínez-Vega, 2017). Estas amenazas explican la importancia del monitoreo de los cambios de usos del suelo.

El estudio y la evolución que se ha producido a lo largo del tiempo de dichos cambios permiten el análisis de procesos medioambientales y de problemas que han podido darse (Lambin y Geist, 2006) y juegan un papel fundamental en el desarrollo económico y social a escala local y regional e, incluso, a escala global. Cambios de usos del suelo locales pueden influir y contribuir al cambio climático global (Meyer y Turner, 1991).

Mediante el uso de matrices de transición o también llamadas matrices de tabulación cruzada (Bell, 1974; Pontius et al., 2004) se pueden obtener valores de cambio de usos del suelo totales, cambio netos, intercambios, ganancias brutas y pérdidas brutas, analizando dos pares de mapas de fechas diferentes y realizando una comparación píxel a píxel. Cuando se trabaja con varios pares temporales cuya escala temporal no es coincidente, es necesario, además de obtener información sobre pérdidas y ganancias totales, analizar con detalle las tendencias cuantificando la intensidad anual de los cambios, con el objeto de que dichos pares sean comparables entre sí. De esta manera, podemos obtener información más detallada acerca de los patrones de cambio entre intervalos de tiempo y aproximarnos a los posibles impulsores del proceso. Hoy en día estos estudios de intensidades de cambio son escasos. Aldwaik y Pontius (2012) presentan la metodología en cuestión y estudian la intensidad de los cambios durante los años 1985, 1991 y 1999 para siete usos del suelo diferentes en un área al norte de Boston, Massachusetts, EE.UU.; Huang et al. (2012) estudian las intensidades en los usos urbanos, agrícolas y naturales durante 1986, 1996 y 2002 en la cuenca del río Juilong, al sureste de China; Raphael et al. (2014) estudian las intensidades en los cambios en siete usos del suelo en los años 1976, 1987 y 2008 en la región de Arusha, Tanzania, y los relacionan con las percepciones y las respuestas de la comunidad a dichos cambios; Rufin et al. (2015) estudian los cambios que se han registrado en pastos y vegetación arbórea entre 1985 y 2012 en Novo Progresso, Brasil, relacionándolos con la fenología; y Farfán et al. (2016) observan las intensidades en trece categorías de cambios de usos del suelo en la Reserva de la Biosfera Sierra de Manantlán, México, los años 2000, 2004 y 2008.

Aldwaik y Pontius (2013) utilizan dicha metodología para observar posibles errores en los mapas que podrían explicar las desviaciones en las intensidades de cambios de usos, aplicándola al mismo área que en su estudio de 2012. Otros autores que usan esta técnica para la detec- 
ción de posibles errores son Pontius et al. (2013), los cuales aplican los análisis de intensidad en el área de Kalimantan central, Indonesia, observando los cambios que se han dado en bosques, pastos y suelos desnudos, y cuantificado cómo pueden afectar los posibles errores en la cartografía a los resultados; o Enaruvbe y Pontius (2015), que utilizan dichos análisis para detectar posibles errores en la clasificación de cuatro usos del suelo al sur de Nigeria entre 1987 y 2002.

La intensidad en el cambio de uso del suelo es definida en este trabajo como la velocidad de un tipo de uso del suelo en cambiar a otro presuponiendo que en el periodo de estudio los cambios hubiesen estado uniformemente distribuidos a través del tiempo. Interesa conocer los usos del suelo que se muestran más dinámicos en diferentes fechas temporales comparándolos con una tasa anual de cambio uniforme. Dicho estudio se realiza independientemente del tamaño que ocupan los diferentes usos del suelo sobre el territorio.

Para esto se aplicará la metodología desarrollada por Aldwaik y Pontius (2012), denominada Intensity Analysis, la cual permite observar de forma jerárquica los cambios de usos del suelo registrados en dos fechas determinadas a nivel de intervalo de tiempo, a nivel de categorías y a nivel de transiciones. Esto permite definir cuáles son los principales procesos de cambio. Además, sirve también para medir la estacionalidad entre dos o más intervalos de tiempo, que se produce cuando el patrón de un intervalo de tiempo es el mismo que el patrón en los otros intervalos de tiempo (Huang et al., 2012). Se aplica a la Comunidad de Madrid con el objeto de observar la intensidad de cambio desde su génesis como Comunidad Autónoma a principios de los años 80 del siglo pasado. Para ello se representarán dichos cambios con el fin de observar la tasa de variación interanual en tres periodos temporales: 1982-1990, 1990-2000 y 2000-2006. Más detalladamente se observará a) en qué intervalos de tiempo la tasa de variación interanual ha sido más rápida o más lenta; b) qué categorías se han mantenido prácticamente inactivas y cuáles han sido más activas; c) si estos patrones mencionados son estables a través de los diferentes intervalos de tiempo; y d) qué transiciones son intensamente evitadas y cuáles son intensamente dirigidas por una categoría de uso del suelo determinada en un intervalo de tiempo determinado.

\section{II. ÁREA DE ESTUDIO}

La Comunidad de Madrid se encuentra localizada en el centro de España. Con una extensión de $8.027 \mathrm{~km}^{2}$ abarca el 1,59\% de la superficie nacional. Se trata de una zona de alta concentración demográfica, con unas densidades de población 8,65 veces mayor que la nacional. Uno de sus rasgos demográficos es la alta concentración de población en la corona metropolitana, es decir, en el término municipal de Madrid y en los términos municipales circundantes (Fig. 1).

La región ha registrado importantes transformaciones territoriales desde mediados de los años 80 y en las últimas dos décadas han acontecido grandes cambios en los usos del suelo, produciéndose como consecuencia un aumento de la artificialización del territorio, relacionada con una masiva expansión de la red de infraestructuras.

A pesar del elevado nivel de antropización de la región, su medio natural posee acreditada diversidad biológica. Una importante superficie regional está bien conservada gracias a diversas figuras de protección, las cuales suponen casi el $75 \%$ del territorio autonómico; al mismo tiempo, más de un $15 \%$ de la superficie total de la región se corresponde con espacios que tienen valores paisajísticos altos o muy altos (Martínez Vega et al., 2007). 


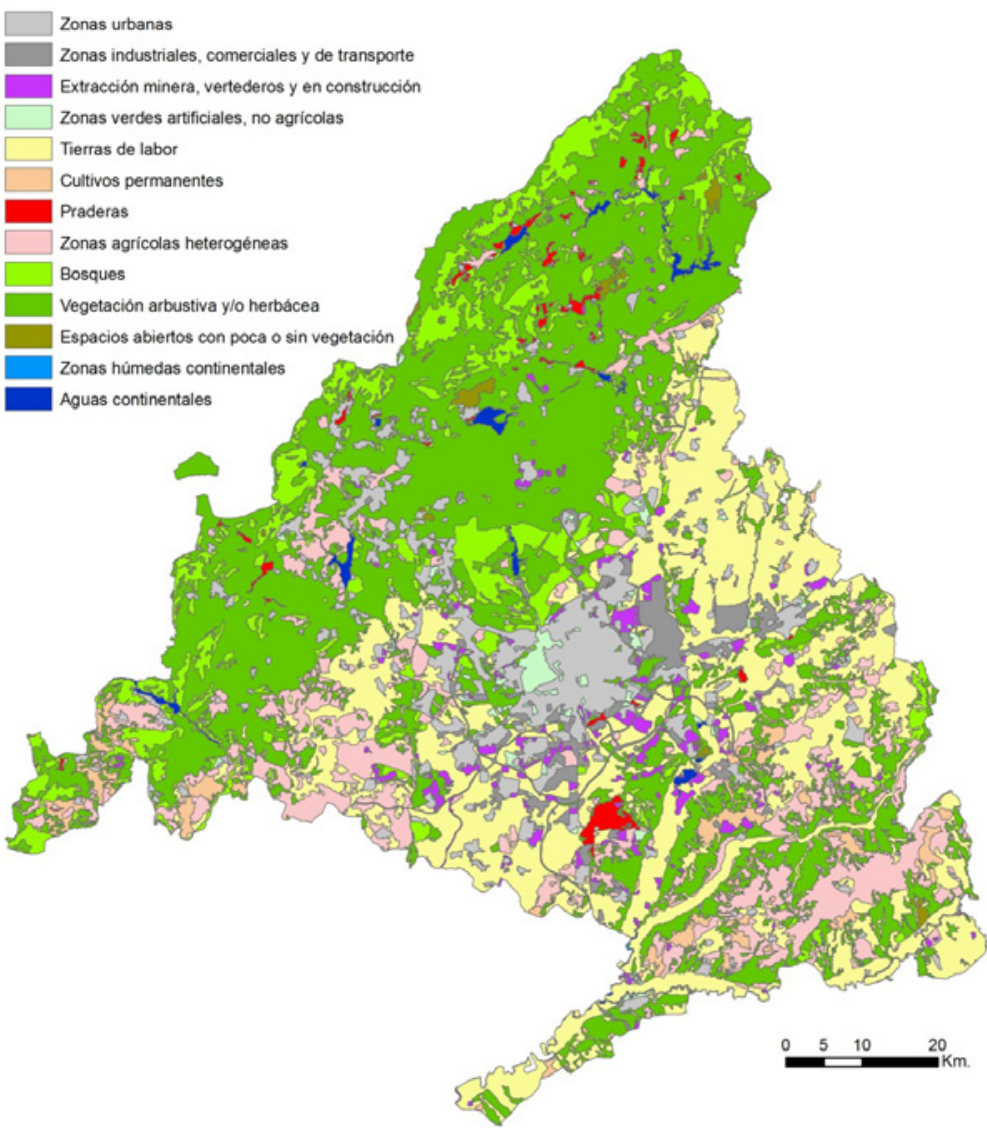

\section{DATOS Y METODOLOGÍA}

Para la realización de los análisis de intensidad del cambio se han utilizado cuatro mapas de usos del suelo, cada uno relativo a un dato temporal (tabla 1). Se escogió la leyenda a nivel 2 del proyecto CORINE Land Cover (CLC) correspondiente en la Comunidad de Madrid con 13 clases de usos del suelo.

Dado que la escala temática y espacial del mapa del año 1982 no coincide con la de los tres mapas CLC, se reclasificó a la leyenda de CLC nivel 3 y se generalizó a una escala de 1:100.000. Para ello se usaron fotografías aéreas, ortofotografías e imágenes de satélite disponibles cercanas a las fecha. Asimismo, se corrigieron errores que se detectaron en la cartografía de CLC al realizar las matrices de tabulación cruzada y que conllevaban a la obtención de cambios de usos del suelo falsos (Gallardo, 2014). Toda la información fue convertida a formato ráster, a un tamaño de píxel de $50 \mathrm{~m}$. 
Tabla 1

FUENTES DE DATOS UTILIZADAS PARA LA REALIZACIÓN DE LOS ANÁLISIS

DE INTENSIDAD DE CAMBIOS DE USOS DEL SUELO

\begin{tabular}{|l|c|c|c|}
\hline Fuente & Año & Escala espacial & Escala temática \\
\hline $\begin{array}{l}\text { Mapa de Vegetación y Usos del Suelo de la Comunidad } \\
\text { de Madrid }\end{array}$ & 1982 & $1: 50.000$ & 63 categorías \\
\hline Mapa CORINE Land Cover & 1990 & $1: 100.000$ & 44 categorías \\
\hline Mapa CORINE Land Cover & 2000 & $1: 100.000$ & 44 categorías \\
\hline Mapa CORINE Land Cover & 2006 & $1: 100.000$ & 44 categorías \\
\hline
\end{tabular}

Los análisis de intensidad en los cambios de usos del suelo, se realizaron entre pares temporales, es decir, entre 1982-1990, 1990-2000 y 2000-2006. Como se observa, dichos intervalos temporales no poseen la misma escala temporal (el primero abarca 8 años; el segundo, 10 años y el tercero, 6 años) y por lo tanto, la información en cuanto a ganancias y pérdidas totales de los cambios que se han sucedido entre un periodo y otro no es comparable. Esta diferencia hace que sea importante realizar un análisis de la tasa de variación interanual, en lugar de considerar únicamente el cambio total.

Basándonos en la metodología de Aldwaik y Pontius (2012 y 2013) se examinaron: a) cómo el tamaño y la tasa o velocidad de cambio varía entre los diferentes intervalos de tiempo (análisis a nivel de intervalo); b) cómo el tamaño y la intensidad de las pérdidas brutas y de las ganancias brutas de cada categoría varía a través de las categorías presentes en cada intervalo de tiempo (análisis a nivel de categoría); y c) cómo el tamaño y la intensidad de las transiciones de cada categoría varía a través de otras categorías que están disponibles para esa transición (análisis a nivel de transición) (Fig. 2).

Figura 2

ESQUEMA JERÁRQUICO UTILIZADO EN LOS ANÁLISIS DE INTENSIDAD

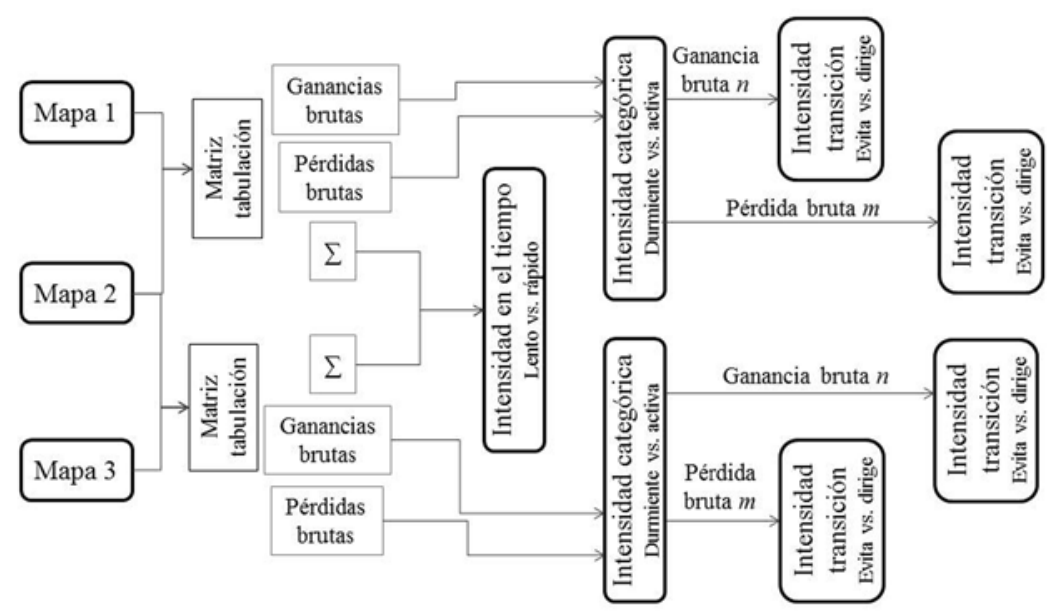

Fuente: Aldwaik y Pontius (2012). 
Específicamente, el análisis a nivel de intervalo examina la tasa de cambio total para cada intervalo de tiempo; se trata de ver cómo el tamaño y la tasa anual de cambio varían en los diferentes intervalos de tiempo; el resultado se compara con una tasa uniforme que existiría si los cambios anuales estuviesen uniformemente distribuidos a través del tiempo.

El análisis a nivel de categoría analiza la intensidad de cada categoría dentro de cada intervalo de tiempo con respecto a la tasa de cambio total que ha revelado el análisis a nivel de intervalo; se examina cada categoría para medir cómo la intensidad y el tamaño de las pérdidas brutas y de las ganancias brutas varía en el espacio; estas intensidades observadas se comparan también con una tasa de cambio anual uniforme que existiría si el cambio de cada intervalo estuviese distribuido uniformemente sobre todo el área de estudio.

Por último, el análisis a nivel de transición estudia la intensidad de la transición con respecto al tamaño de las categorías y en relación con los resultados obtenidos de los análisis a nivel de categoría; se considera cada transición de forma particular para examinar cómo el tamaño y la intensidad de dicha transición varía entre las categorías disponibles para esa transición; cuando una categoría gana o pierde, este análisis puede identificar qué otras categorías están siendo intensivamente evitadas o dirigidas, comparando la intensidad observada de cada transición con una intensidad uniforme que existiría si la transición estuviese distribuida uniformemente entre las categorías disponibles para dicha transición.

El nivel de intervalo está definido por las siguientes ecuaciones:

$$
\begin{aligned}
& S t=\frac{\text { Superficie de cambio durante el intervalo } Y / \text { Superficie total }}{\text { Duración del intervalo } Y} \times 100 \\
& U=\frac{\text { Superficie de cambio durante todos los intervalos } / \text { Superficie total }}{\text { Duración de todos los intervalos }} \times 100
\end{aligned}
$$

La primera ecuación define el porcentaje de cambio anual del área de estudio para cada intervalo de tiempo, mientras que la segunda ecuación define el valor de cambio anual uniforme, el cual se produce cuando se distribuye el cambio total de manera uniforme durante los intervalos, desde el primer punto en el tiempo hasta el último.

Si la intensidad del intervalo es inferior a la del periodo dado, el cambio que se ha producido es relativamente lento, mientras que si es superior, se considera que es relativamente rápido.

El nivel de categoría está definido por:

$$
\begin{aligned}
G t j & =\frac{\text { Superficie de ganancia bruta de categoría } j \text { en intervalo } Y / \text { duración del intervalo } Y}{\text { Superficie de categoría } \text { jen la última fecha del intervalo } Y} \times 100 \\
L t i & =\frac{\text { Superficie de pérdida bruta de categoría } \text { i en intervalo } Y / \text { duración del intervalo } Y}{\text { Superficie de la categoría i en la primera fecha del intervalo } Y} \times 100
\end{aligned}
$$

Estas dos ecuaciones nos indican qué categorías son las que están inactivas y cuáles son las más activas para un intervalo de tiempo dado, así como la intensidad de ganancia y/o pérdida anual de cada categoría.

Una categoría se puede considerar activa, en cuanto a la intensidad, cuando la ganancia o pérdida de dicha categoría es superior al promedio anual, o inactiva si es inferior al promedio anual. El método nos informa también si el patrón de dicha categoría es estable en el tiempo 
en términos de intensidad de ganancias y de pérdidas: si la categoría es activa o inactiva en todos los intervalos de tiempo, el patrón será estable; si es activa en un intervalo pero inactiva en otro, el patrón no será estable.

El nivel de transición está definido por el análisis de transición de una categoría a otra en base a las ganancias brutas y a las pérdidas brutas:

$$
\begin{aligned}
& \text { Rtin }=\frac{\text { Superficie de transición de i a n en intervalo } Y / \text { duracion del intervalo } Y}{\text { Superficie de categoría i en la fecha inicial del intervalo } Y} \times 100 \\
& W t n=\frac{\text { Superficie de ganancia bruta de categoría } n \text { en intervalo } Y / \text { duración del intervalo } Y}{\text { Superficie que no es categoría n en la fecha inicial del intervalo } Y} \times 100 \\
& Q t m j=\frac{\text { Superficie de transición de m a j en intervalo } Y / \text { duración del intervalo } Y}{\text { Superficie de la categoría } \text { jen la fecha final del intervalo } Y} \times 100 \\
& V t m=\frac{\text { Superfice de pérdida bruta de categoria } m \text { en intervalo } Y / \text { duración del intervalo } Y}{\text { Superficie que no es categoría } m \text { en la fecha final del intervalo } Y} \times 100
\end{aligned}
$$

Las dos primeras ecuaciones aquí presentes identifican qué otras categorías están siendo excluidas y cuáles no lo son debido a la ganancia de la categoría $n$ en el intervalo dado. Las dos últimas ecuaciones identifican qué otras categorías están siendo excluidas y cuáles no debido a la pérdida de la categoría $m$ en el intervalo dado.

Si la intensidad anual de transición desde una categoría es superior a la intensidad uniforme de transición, se considera que dicha transición utiliza sistemáticamente dicha categoría, mientras que si es inferior, la evita.

$\mathrm{Al}$ igual que en el caso de la intensidad a nivel de categoría, se puede determinar si dicha transición es estable a lo largo del periodo de estudio, comparando dicha categoría en los diferentes intervalos de tiempo; si la excluye o no la excluye en todos los intervalos será estable, mientras que si la excluye en un intervalo y en otro no, no será estable.

La metodología de análisis sigue el esquema presentado en la figura 2. Los resultados se presentan a nivel 2 de CLC. En cuanto a la intensidad a nivel de transición, dado que existen hasta 42 transiciones diferentes para el nivel 2 de CLC en los tres periodos en total, se muestran únicamente los resultados de las dos transiciones que se han considerado como las más interesantes, esto es, hacia zonas urbanas y desde tierras de labor.

\section{RESULTADOS}

Los resultados se obtuvieron a nivel de intervalo, a nivel de categoría y a nivel de transición para cada uno de los tres periodos señalados, 1982-1990, 1990-2000 y 2000-2006.

\section{IV.1. A nivel de intervalo}

La tabla 2 detalla la intensidad a nivel de intervalo para los tres periodos, en porcentajes con respecto al total.

Se observa que el porcentaje de cambio ha sido mayor en el periodo comprendido entre 1982 y 1990 que en los dos últimos periodos. Si se observa el cambio anual, este intervalo coincide también con el de mayor intensidad, estando por encima de la velocidad uniforme del cambio $(1,03)$, lo que quiere decir que, a pesar de que la duración de este periodo es menor que la duración del segundo periodo, la intensidad de los cambios ha sido mayor; 
dicho cambio anual se ha producido, de hecho, bastante rápido, mientras que en los años 1990-2000 y 2000-2006, el cambio se ha producido relativamente lento, con respecto a la velocidad uniforme de cambio.

Tabla 2

ANÁLISIS DE INTENSIDAD A NIVEL DE INTERVALO, EN PORCENTAJES CON RESPECTO AL TOTAL, A NIVEL 2 DE CLC

\begin{tabular}{|c|c|c|c|c|}
\hline Intervalo & Cambio observado & Cambio anual & $\begin{array}{c}\text { Velocidad uniforme } \\
\text { de cambio }\end{array}$ & Cambio uniforme \\
\hline $1982-1990$ & 12,80 & 1,60 & 1,03 & 8,20 \\
\hline $1990-2000$ & 6,61 & 0,66 & 1,03 & 10,25 \\
\hline $2000-2006$ & 5,19 & 0,87 & 1,03 & 6,15 \\
\hline
\end{tabular}

El ratio de cambio no es uniforme a lo largo del periodo. Si la velocidad de cambio se hubiese mantenido constante para todo el conjunto temporal, esto es, a una tasa de cambio de 1,03 , se deberían haber registrado valores de cambio más bajos para el periodo 19821990 (8,20\% con respecto a 12,80\%), valores bastante más altos para el periodo 1990-2000 $(10,25 \%$ con respecto $6,61 \%)$ y valores relativamente más altos para el periodo $2000-2006$ $(6,15 \%$ con respecto $5,19 \%)$.

\section{IV.2. A nivel de categoría}

Los resultados obtenidos a nivel 2 de CLC para los tres intervalos estudiados a nivel de categoría se muestran en la figura 3. El eje horizontal representa la intensidad de cambio anual durante el intervalo correspondiente, en porcentajes.

La línea vertical discontinua nos muestra la intensidad de cambio anual uniforme para todo el área de estudio; si la barra de una categoría, ya sea de ganancias brutas o de pérdidas brutas, se localiza a la izquierda de la línea uniforme, quiere decir que el cambio de dicha categoría se presenta de manera relativamente inactiva (o durmiente), mientras que si la barra se extiende a la derecha de dicha línea uniforme, el cambio es relativamente activo para esa categoría ${ }^{1}$.

Esta figura permite observar la intensidad de actividad de las diferentes categorías, independientemente del tamaño que ocupan dichas categorías sobre el territorio.

Las zonas de extracción minera, vertederos y en construcción experimentan ganancias de manera muy intensa en comparación con otras categorías, ya que se extienden ampliamente a la derecha de la línea uniforme; dicha intensidad se produce en los tres intervalos de tiempo, lo cual indica que esta categoría es estacionaria ${ }^{2}$, y experimenta una intensidad creciente en el tiempo.

1 Aldwaik y Pontius (2012) definen las categorías de usos del suelo que se localizan a la izquierda de la línea de cambio uniforme como dormant y las que se localizan a la derecha como active. En este estudio se ha referido a ellas como durmientes y activas respectivamente, teniendo en cuenta que una categoría considerada como durmiente es una categoría que ha experimentado cambios (pérdidas y/o ganancias) pero con una intensidad o velocidad de cambio inferior a la intensidad de cambio anual uniforme.

2 Aldwaik y Pontius (2012) definen que una categoría es stationary cuando la intensidad de cambio que experimenta dicha categoría posee el mismo patrón en todos los intervalos de tiempo. En este estudio se ha hecho una traducción literal del término, denominando este proceso como estacionario. 
Las zonas industriales, comerciales y de transporte, y las zonas verdes artificiales también experimentan ganancias de manera más intensa que otras categorías en los tres periodos de tiempo. Es, esta última, la que mayor intensidad presenta en el periodo 1982-1990.

Figura 3

ANÁLISIS DE INTENSIDAD DE CAMBIO ANUAL POR CATEGORÍAS, A NIVEL 2 DE CLC, EN GANANCIAS Y PÉRDIDAS BRUTAS, EN PORCENTAJES. A) PERIODO 1982-1990; B) PERIODO 1990-2000; C) PERIODO 2000-2006. LA LÍNEA RECTA CORRESPONDE A LA LÍNEA DE UNIFORMIDAD, EN CADA PERIODO (A: LU 1,60; B: LU 0,66; C: LU 0,87)
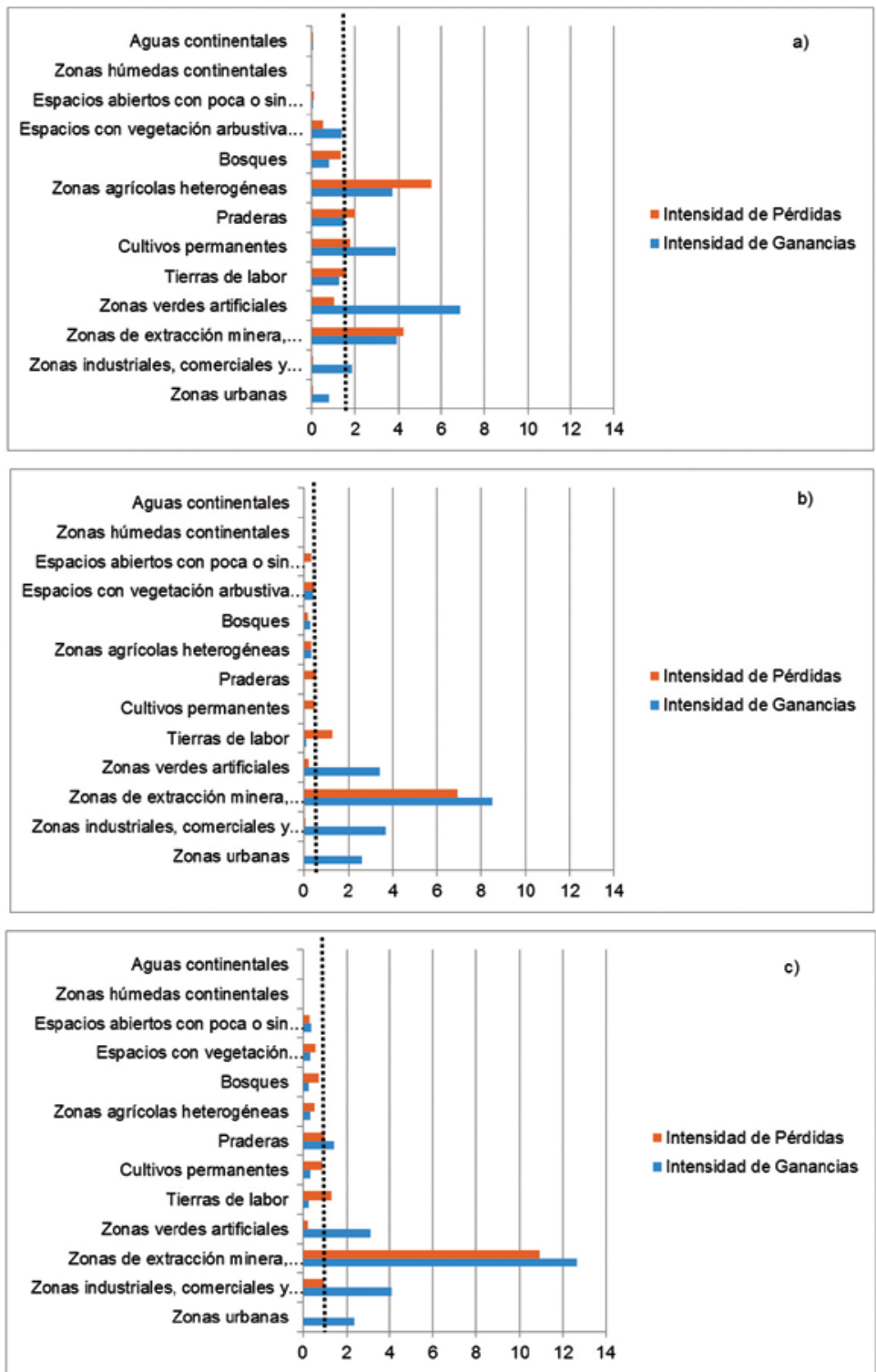
En cuanto a las pérdidas, la categoría de extracción minera, vertederos y en construcción se presenta con mayor intensidad con respecto al resto de categorías en los tres periodos de tiempo, indicando que dicha categoría es estacionaria, tanto en intensidad de ganancias como en intensidad de pérdidas. Es, además, la que mayor intensidad de pérdidas posee en los periodos 1990-2000 y 2000-2006, mientras que en el primer periodo, 1982-1990, ésta se corresponde con las zonas agrícolas heterogéneas.

Existen categorías que se mantienen durmientes con respecto al resto. Éstas son las que se encuentran a la izquierda de la línea de uniformidad. Para el periodo 1982-1990 destaca la inactividad, en cuanto las ganancias, de las tierras de labor y los bosques y, en cuanto a las pérdidas, de los espacios con vegetación arbustiva y/o herbácea. En el periodo 1990-2000 destaca la inactividad, en cuanto a las ganancias, de las tierras de labor (y lo mismo ocurre en el periodo 2000-2006) y, en cuanto a las pérdidas, la categoría de bosques; para el periodo 2000-2006 la mayor inactividad de pérdidas se da en los espacios abiertos con poca o sin vegetación.

Las aguas continentales y las zonas húmedas no presentan ninguna ganancia o pérdida, ya que no han experimentado ningún cambio durante los tres intervalos de tiempo que abarca este estudio.

\section{IV.3. A nivel de transición}

Las figuras 4 y 5 presentan los resultados de las dos transiciones que se han considerado como las más interesantes, esto es: los usos del suelo que han pasado a ser zonas urbanas (Fig. 4) y las tierras de labor que han pasado a ser otros usos (Fig. 5).

El eje horizontal representa la intensidad de transición anual, como porcentaje de la categoría de ganancia de zonas urbanas (en el primer caso), y como porcentaje de la categoría de pérdida de tierras de labor (en el segundo caso). La línea horizontal discontinua muestra la transición anual uniforme; las barras que están a la izquierda de dicha línea horizontal representan categorías que tienden a evitar dicha transición, mientras que las que están situadas a la derecha son las categorías que dirigen esa transición.

Las zonas urbanas crecen más intensamente desde las zonas de extracción minera, vertederos y zonas en construcción, u otros usos artificiales, que desde usos agrícolas. Dicha ganancia, dirigida especialmente por la toma de zonas de extracción minera, vertederos y en construcción, se muestra en los tres periodos de estudio, lo que quiere decir que este proceso es estacionario.

En el intervalo 1982-1990, las zonas verdes artificiales, seguidas de los prados y praderas, son los usos del suelo que se muestran más intensificados en cuanto a su sustitución por zonas urbanas. Entre 1990-2000, destacan los prados y praderas y las tierras de labor, aunque muy por debajo de las zonas de extracción minera, vertederos y en construcción. En el último periodo de estudio, 2000-2006, son las zonas industriales, comerciales y de transporte, así como los prados y praderas, las que se muestran con más intensidad, pero, también, de manera bastante menos enérgica que las zonas de extracción minera, vertederos y áreas en construcción.

Los usos del suelo que están representados a la izquierda de la línea de uniformidad, pueden considerarse, en cuanto a la intensidad de cambio, que eluden o evitan su transición a zonas urbanas ${ }^{3}$.

3 Aldwaik y Pontius (2012) definen a las categorías que están localizadas a la izquierda de la línea de uniformidad como categorías que se comportan de forma avoid en cuanto al cambio. Se ha hecho una traducción literal del término, denominándolas como categorías que eluden o evitan el cambio. 
ANÁLISIS DE INTENSIDAD DE CAMBIO ANUAL POR TRANSICIÓN HACIA ZONAS URBANAS, A NIVEL 2 DE CLC EN PORCENTAJES. A) PERIODO 1982-1990; B) PERIODO 1990-2000; C) PERIODO 2000-2006. LA LÍNEA RECTA CORRESPONDE A LA LÍNEA DE UNIFORMIDAD, EN CADA PERIODO (A: LU 0,04; B: LU 0,19; C: LU 0,21)
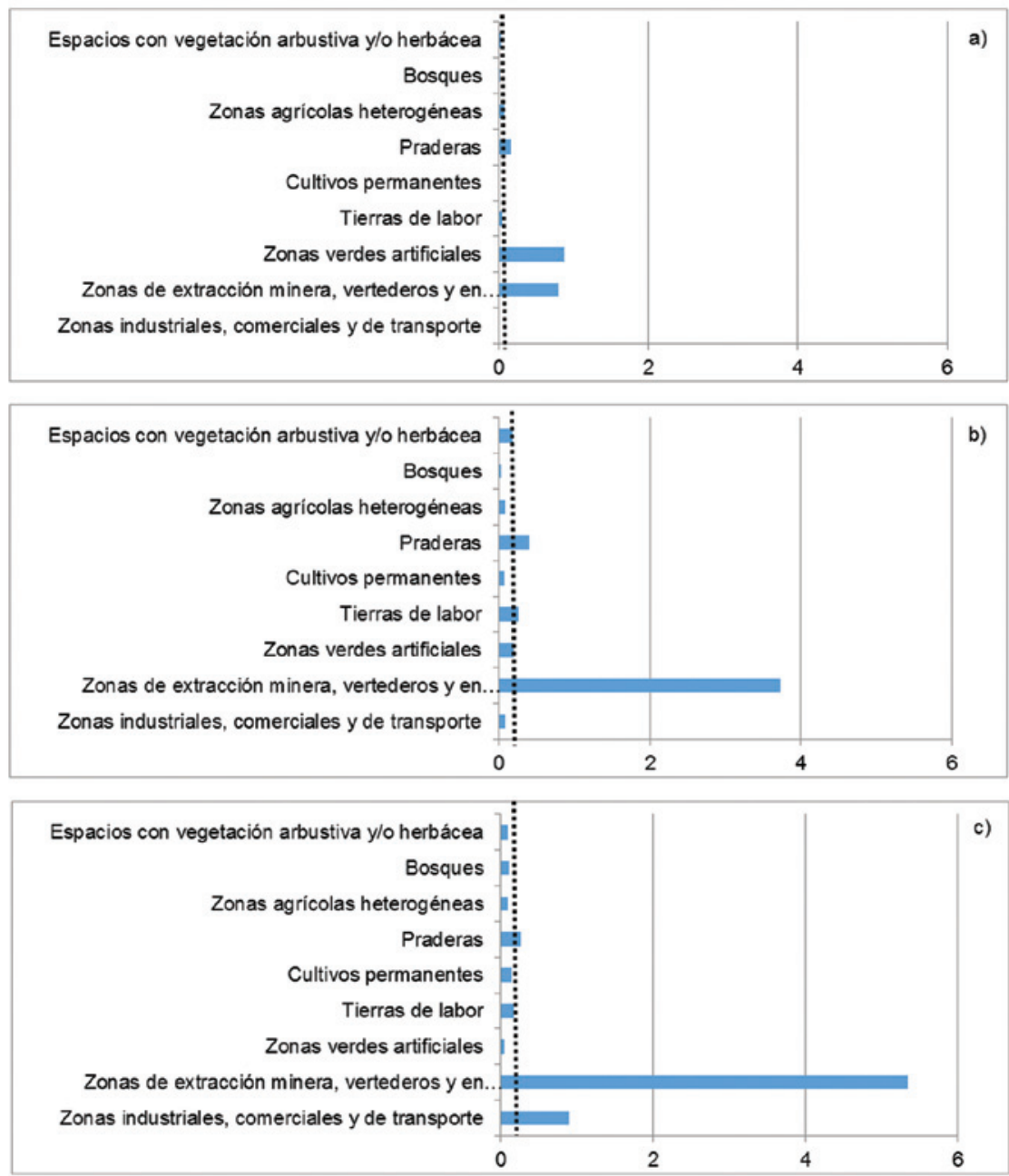

La figura 5 muestra la intensidad de transición que se ha dado desde las tierras de labor hacia otros usos del suelo en los tres periodos estudiados. Así, se observa que las transiciones que se han producido desde las tierras de labor, cuando este uso del suelo experimenta pérdidas, tiende a ser sustituido, de forma intensiva, por zonas agrícolas heterogéneas, cultivos permanentes, zonas de extracción minera, vertederos y en construcción, y zonas industriales, comerciales y de transporte, en el periodo 1982-1990; por zonas de extracción minera, vertederos y en construcción, seguido de zonas industriales, comerciales y de transporte, y zonas urbanas entre 1990-2000; y por zonas de extracción minera, vertederos y en construc- 
ción y, en menor medida, por zonas industriales comerciales y de transporte, zonas verdes artificiales, y zonas urbanas. La categoría de zonas de extracción minera, vertederos y en construcción y las zonas industriales, comerciales y de transporte se comportan de manera estacionaria.

Los usos del suelo que se mantienen a la izquierda de la línea de uniformidad pueden considerarse que suelen rehuir las tierras de labor.

Figura 5

ANÁLISIS DE INTENSIDAD DE CAMBIO ANUAL POR TRANSICIÓN DESDE TIERRAS DE LABOR, A NIVEL 2 DE CLC, EN PORCENTAJES. A) PERIODO 1982-1990; B) PERIODO 1990-2000; C) PERIODO 2000-2006. LA LÍNEA RECTA CORRESPONDE A LA LÍNEA DE UNIFORMIDAD, EN CADA PERIODO (A: LU 0,63; B: LU 0,48; C: LU 0,41)
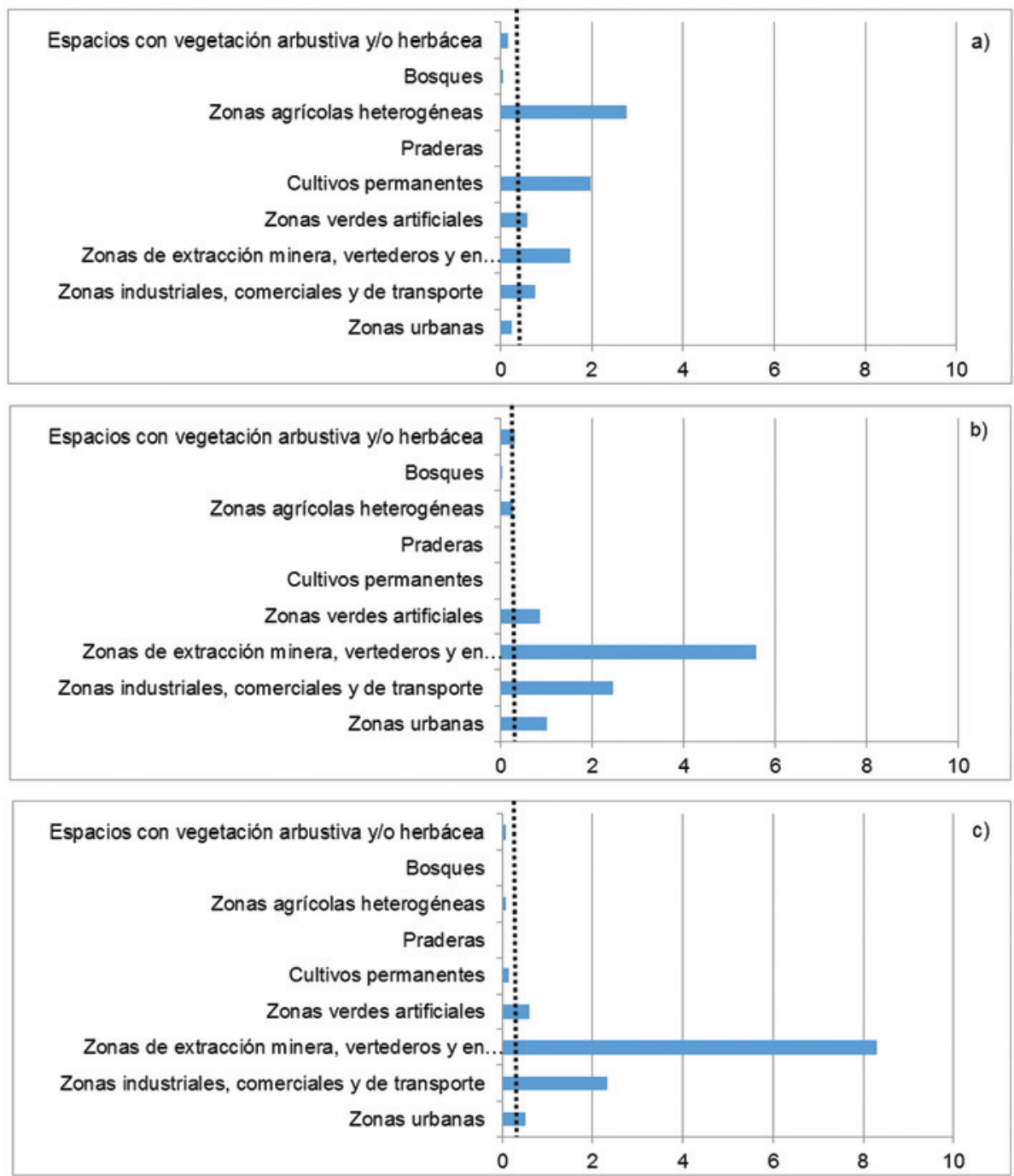
En resumen, puede decirse que el periodo de cambio más intenso se ha producido durante 1982-1990, seguido del comprendido por los años 2000 y 2006. Las ganancias más intensas se han producido en las zonas de extracción minera, verteros y en construcción (destaca en los tres periodos estudiados), seguida de las zonas industriales, comerciales y de transporte y las zonas verdes artificiales. En cuanto a las pérdidas, el uso del suelo que ha registrado más intensidad ha sido también las zonas mineras, vertederos y en construcción. Dentro de esta categoría, el uso del suelo que está provocando estos resultados es generado, en realidad, por las zonas en construcción, las cuales aparecen como un paso previo a la ocupación por parte de usos urbanos, industriales, comerciales o de transporte. Los bosques destacan por su inactividad o estabilidad.

\section{DISCUSIÓN Y CONCLUSIONES}

Los datos obtenidos en cuanto a la intensidad en los cambios de usos del suelo están relacionados con la recuperación económica que se produce con la entrada de España en la Unión Europea en 1986 y que posibilita la intensificación de la reticulación y la introducción de la filosofía de las hiperconexiones en las grandes ciudades (de Santiago, 2008). La región de Madrid representó en este sentido un enclave estratégico para la instalación de nuevas industrias, actividades y equipamientos terciarios, junto a la promoción urbana.

Los cambios experimentados están sustentados fundamentalmente en el crecimiento de las superficies artificiales en detrimento de las superficies agrícolas, siendo el uso más dinámico e intenso los cambios de y hacia superficies en construcción. Ha sido la evolución de las infraestructuras de transporte la verdadera base material de la transformación de la Comunidad de Madrid en estas décadas pasadas (Serrano Cambronero, 2002; López de Lucio, 2003), vinculadas al interés, por parte de las administraciones regionales, por construir infraestructuras de gran capacidad para el transporte privado (Fernández Güell, 2008; Valenzuela, 2010), causando la rápida ocupación de las áreas próximas a la metrópoli. La ocupación de suelo por autopistas nuevas y por la ampliación aeroportuaria explica el 80\% de la formación de suelo por equipamientos entre 1980 y 2005 (Naredo y García Zaldivar, 2008). Tal y como comenta Verburg et al. (2004), la accesibilidad, medida en distancia y tiempo de transporte, es uno de los factores que más influencian los cambios de usos del suelo, especialmente en regiones altamente urbanizadas.

Se ha incentivado el abandono de suelos labrados que han sido invadidos no sólo por usos artificiales sino también por vegetación arbustiva. Hoy en día, el espacio agrario madrileño está sometido a fuertes presiones urbanas e industriales que reducen su superficie y repercuten en su alto nivel de degradación. Su dimensión económica es escasa, a pesar de tener el incentivo de localizarse junto a un gran mercado urbano (Méndez, 2001). El sector forestal no tiene especial relevancia frente a otras ramas de la actividad económica; su importancia radica en el papel medioambiental y de esparcimiento que conforman y se ha mantenido más o menos estable durante todos estos años.

El desarrollo urbano y de infraestructuras que se ha venido desarrollando en este último periodo ha tenido sus impactos negativos, especialmente en la esfera medioambiental y de sostenibilidad. El trazado de infraestructuras de transporte ha ocasionado una fragmentación del medio natural. Se ha sobredimensionado el suelo urbanizable y el parque de vivien- 
das secundarias y/o desocupadas, produciendo una superdestrucción de los asentamientos, los ecosistemas y los paisajes precedentes. Esto ha supuesto un grave deterioro ecológico (Naredo, 2009). La presencia de esta gran área metropolitana se traduce en una exponencial producción de residuos urbanos e industriales, en la sobreexplotación de algunos recursos naturales y en la presión del ocio sobre los espacios naturales protegidos. A pesar de todo ello, la región sigue conservando un importante número de espacios naturales de incuestionable valor ecológico, bajo diversas figuras de protección, si bien amenazados por el incremento del suelo artificial señalado.

\section{AGRADECIMIENTOS}

La autora fue beneficiaria de una estancia corta en la Universidad de Clark, EE.UU, financiada a través del programa Predoc Jae-CSIC. Agradece al profesor Gil Pontius su inestimable ayuda y el tiempo invertido.

\section{REFERENCIAS}

ALDWAIK, S.Z. y PONTIUS, R.G. Jr. (2012): «Intensity analysis to unify measurements of size and stationarity of land changes by interval, category and transition». Landscape and Urban Planning, $\mathrm{n}^{\circ}$ 106, 103-114.

ALDWAIK, S.Z. y PONTIUS, R.G. Jr. (2013): «Map errors that could account for deviations from a uniform intensity of land change». International Journal of Geographical Information Sciences, vol. 7, $\mathrm{n}^{\circ}$ 9, 1717-1739.

ALIG, R.J., KLINE, J.D. y LICHTESTEIN, M. (2004): «Urbanization on the US landscape: looking ahead in the $21^{\text {st }}$ century». Landscape and Urban Planning, $\mathrm{n}^{\circ} 69,219-234$.

BELL, E.J. (1974): «Markov analysis of land use change - an application of stochastic processes to remotely sensed data». Socio-Economic Planning Sciences, $\mathrm{n}^{\circ}$ 8, 311-316.

CHEN, X. (2002): «Using remote sensing and GIS to analyse land cover change and its impacts on regional sustainable development». International Journal of Remote Sensing, $n^{\circ} 23,107-124$.

DE SANTIAGO, E. (2008): «Madrid “ciudad única” (II). La explosión urbana en la región madrileña y sus efectos colaterales». Urban, $\mathrm{n}^{\circ} 13,138-164$.

ENARUVBE, G.O. y PONTIUS, R.G. Jr. (2015): «Influence of classification errors on intensity analysis of land changes in southern Nigeria». International Journal of Remote Sensing, $\mathrm{n}^{\mathrm{O}} 36,244-261$.

FARFÁN, M., RODRIGUEZ-TAPIA, G. y MAS, J.F. (2016): «Análisis jerárquico de la intensidad de cambio de cobertura/uso del suelo y deforestación (2000-2008) en la Reserva de la Biosfera Sierra de Manantlán, México». Investigaciones Geográficas, Boletín del Instituto de Geografía, UNAM, nº 90, 89-104.

FERNÁNDEZ GÜELL, J.M. (2008): «Condiciones para abordar la ordenacion territorial de una región urbana dinámica: el caso de la Comunidad de Madrid», en I Congreso de Urbanismo y Ordenacion del Territorio. Bilbao, C.y.P. Colegio de Ingenieros de Caminos, 1-21. Disponible en http://www.ciccp.es/biblio_digital/Urbanismo_I/inicio.htm 
GAGO, C., SERRANO, M. y ANTÓN, F.J. (2004): «Repercusiones de las carreteras orbitales de la Comunidad de Madrid en los cambios de usos del suelo». Anales de Geografía, $\mathrm{n}^{\mathrm{o}} 24,145-167$.

GALlARDO, M. (2014): Cambios de usos del suelo y simulación de escenarios en la Comunidad de Madrid. Análisis de tres décadas de transformación territorial y proyección futura. Tesis doctoral. Madrid. Universidad Complutense de Madrid. Disponible en http://eprints.ucm.es/25253/1/T35351.pdf

GALLARDO, M., GÓMEZ, I., VILLAR, L., MARTINEZ-VEGA, J., y MARTIN, M.P. (2016): «Impacts of future land use/land cover on wildfire ocurrence in the Madrid region (Spain)». Regional Environmental Change, vol. 16, $\mathrm{n}^{\circ}$ 4, 1047-1061.

GALLARDO, M. y MARTÍNEZ-VEGA, J. (2017): «Future land use change dynamics in Natural Protected Areas. Madrid region case study» en Proceedings of the $3^{\text {rd }}$ International Conference on Geographical Information Systems Theory, Applications and Management (GISTAM), (Ragia, L., Rocha, J.G. y Laurini, R., eds.). Portugal, Scitepress, 370-377.

GARCÍA PALOMARES, J.C. y GUTIÉRREZ PUEBLA, J. (2007): «La ciudad dispersa: cambios recientes en los espacios residenciales de la Comunidad de Madrid». Anales de Geografía, n 47, 45-67.

HEWITT, R., PERA, F. y ESCOBAR, F. (2016): «Cambios recientes en la ocupación del suelo de los parques nacionales españoles y su entorno». Cuadernos Geográficos, vol. $55, \mathrm{n}^{\circ} 2,46-84$.

HUANG, J., PONTIUS, R.G. Jr., LI, Q. y Zhang, Y. (2012): «Use of intensity analysis to link patterns with processes of land change from 1986 to 2007 in a coastal watershed of woutheast China». Applied Geography, n 34, 371-384.

JANSEN, L.J.M. (2006): «Harmonization of land use class sets to facilitate compatibility and comparability of data across space and time». Journal of Land Use Science, $\mathrm{n}^{\circ} 1$, 127-156.

LAMBIN, E.F. y GEIST, H.J. (Eds.). (2006): Land-use and land-cover change. Local processes and global impacts. Berlin Heildelberg, Springer.

LÓPEZ DE LUCIO, R. (2003): «Transformaciones territoriales recientes en la región urbana de Madrid». Urban, $n^{\circ}$ 9, 124-161.

MALLINIS, G., EMMANOLOUDIS, D., GIANNAKOPOULOS, V., MARIS, F. y KOUTSIAS, N. (2011): «Mapping and interpreting historical land cover/land use changes in a Natura 2000 site using earth observational data: The case of Nestos delta, Greece». Applied Geography, $\mathrm{n}^{\circ}$ 31, 312-320.

MARTÍNEZ VEGA, J., ROMERO CALCERRADA, R., y ECHAVARRÍA, P. (2007): «Valoración paisajística y ecológica de la Comunidad de Madrid: su integración en un indice sintético de riesgo de incendios forestales». Revista de Teledetección, $\mathrm{n}^{\circ}$ 28, 43-60.

MÉNDEZ, R. (2001): «Transformaciones económicas y reorganización territorial en la región metropolitana de Madrid». EURE, vol. 27, nº 80, 141-161

MEYER, W.B. y TURNER II, B.L. (1991): Changes in land use and land cover: A Global perspective. Cambridge, Cambridge University Press.

NAREDO, J.M. y GARCÍA-ZALDÍVAR, R. (2008): Estudio sobre la ocupación del suelo por usos urbano-industriales, aplicado a la Comunidad de Madrid. Madrid, UPM-Ministerio de Medio Ambiente. 
NAREDO, J.M. (2009): «La cara oculta de la crisis. El fin del boom inmobiliario y sus consecuencias». Revista de Economía Crítica, $\mathrm{n}^{\circ}$ 7, 313-340.

PEÑA, J., BONET, A., BELLOT., J., SÁNCHEZ., J.R., EISENHULTH, D., HALLET, S., ALEDO, A. (2007): «Driving forces of land-use change in a cultural landscape of Spain», en Modelling land-use change. Progress and applications (Koomen, E., Stillwell, J., Bakema, A. y Scholten, H.J, Eds). Springer Netherlands. 97-115.

PÉREZ-HUGALDE, C., ROMERO-CALCERRADA, R., DELGADO-PÉREZ, P., y NOVILLO, C. J. (2011): «Understanding land cover change in a Special Protection Area in Central Spain through the enhanced land cover transition matrix and a related new approach». Journal of Environmental Management, vol. 92, nº 4, 1128-1137.

PLATA ROCHA, W., GÓMEZ DELGADO, M., y BOSQUE SENDRA, J. (2009): «Cambios de usos del suelo y expansión urbana en la Comunidad de Madrid 1990-2000». Scripta Nova, num. 13(293).

PONTIUS, R.G. Jr., SHUSAS, E. y McEchern, M. (2004): «Detecting important categorical land changes while accounting for persistence». Agriculture, Ecosystems and Environment, $\mathrm{n}^{\mathrm{o}} 101,251-268$.

PONTIUS., R.G. Jr., GAO, Y., GINER, N.M., KOHAYAMA, T., OSAKI, M. y HIROSE, K. (2013): «Design and interpretation of intensity analysis illustrated by land change in Central Kalimantan». Indonesia Land, vol. 2, n 3, 351-369.

RAPHAEL, L., HAMBATI, H. y ATO ARMAH, F. (2014): «An intenisty analysis of landuse and land-cover change in Karatu District, Tanzania: community perceptions and coping strategies». African Geographical Review, n 33, 150-173.

RUIZ BENITO, P., CUEVAS, J. A., BRAVO DE LA PARRA, R., PRIETO, F., GARCÍA DEL BARRIO, J. M., y ZAVALA, M. A. (2010): «Land use change in a Mediterranean metropolitan region and its periphery: assessment of conservation policies through CORINE Land Cover data and Markov models». Forest Systems, vol. 19, n 3, 315-328.

RURFIN, P., MÜLLER, H., PLUGMACHER, D. y HOSTERT, P. (2015): «Land use intensity trajectories on Amazonian pastures derived from Landsat time series». International Journal of Applied Earth Observation and Geoinformation, $\mathrm{n}^{\circ}$ 41, 1-10.

SERRANO CAMBRONERO, M. (2002): «Influencia de las carreteras en el desarrollo urbano de la Comunidad de Madrid en el periodo 1987-1997». Anales de Geografía de la Universidad Complutense. Vol. Extraordinario, 459-469.

VALENZUELA, M. (2010): «La planificación territorial de la región metropolitana de Madrid. Una asignatura pendiente». Cuadernos Geográficos, nº 47, 95-129.

VERBURG, P.H., OVERMARS, K.P. y WITTE, N. (2004): «Accesibility and land-use patterns at the forest fringe in the northeastern part of the Philippines». Geographical Journal, $\mathrm{n}^{\mathrm{o}} 170,238-255$.

ZHANG, H., QI, Z.F., YE, X.Y., CAI, Y.B., MA, W.C., y CHEN, M.N. (2013): «Analysis of land use/land cover change, population shift, and their effects on spatiotemporal patterns of urban heat islands in metropolitan Shanghai, China». Applied Geography, n 44, 121 133. 